\title{
Relanzamiento del Mercosur
}

\author{
Enrique Deigodo \\ Embajodor de lo Repúblico del Uruguoy
}

$\mathrm{L}$ a idea central de la ponencia se vincula con la identificación de una agenda que compren da los desafíos que tiene el Mercosur al inicio del nuevo milenio.

En mi caso particular, nos vamos a concentrar en lo que los presidentes de los estados miembros del bloque han denominado como el relanzamiento del Mercosur.

La perspectiva estará dirigida a la agenda interna del Mercosur; los retos que tiene para en el futuro inmediato para acelerar el proceso de integración que nace con el tratado de Asunción en el año 1991.

La ponencia se va a dividir en dos tramos, una primera con una presentación de la realidad institucional para después abocarnos a los temas que integran la agenda futura del Mercosur.

\section{Sistema institucional, principios e instrumentos}

El acto fundacional del Mercosur se constituye con el tratado de Asunción que se suscribe en el mes de marzo del año 1991, donde se establece un acuerdo marco de duración indefinida con un esquema institucional transitorio que tiene por objetivo la conformación de un mercado común y es de vocación regional.

La decisión de constituir un mercado común en un plazo determinado, indica cuál es el límite de la voluntad política de los estados miembros.

El objetivo no es en principio alcanzar una unión económica ni una unión política.Por otro lado, es un acuerdo esquemático; una característica que nos lleva a definirlo como un convenio marco.

En otras palabras, los instrumentos que se indican en el mismo para alcanzar el objetivo del tratado, deben ser desarrollados en otros convenios y actos jurídicos vinculantes.
Los principios que se recogen en el acucrdo son los clásicos en todo tratado de integración.

- La reciprocidad de derechos y obligaciones entre los cstados partes (art.2);

- La no discriminación entre los mismos (art.8 inciso d);

- Las diferencias puntuales de ritmo en el programa comercial para paraguay y Uruguay (art.6);

- El trato nacional para productos provenientes de cualquier estado parte (art.7):

- La transparencia en la coordinación de politicas nacionalcs para asegurar condiciones equitativas de comercio con terceros (art.4):

- El principio pacta sunt servanda relacionado con los perjuicios que puedan surgir de las negociaciones comerciales externas (art. 8 incisos a y b);

Por su lado, los instrumentos para alcanzar la meta de un mercado común están definidos en el art. 5 del tratado de Asunción.

El artículo define cuatro instrumentos:

1. Un programa de liberación comercial, que se encuentra desarrollado en el anexo l, e incorporado en el acuerdo de alcance parcial $n^{\circ} 18$.

2. La coordinación cle politicas macroeconómicas que debe ser realizada en forma convergente con los programas de desgravación arancelaria.

3. La eliminación de restricciones no arancelarias.

4. La definición del arancel externo común; y la adopción de acuerdos sectoriales.

En dos etapas, una con el tratado de Asunción y otro a partir de la suscripción del tratado de Ouro Preto. se logra conformar la zona de libre comercio.

A partir del 31 de diciembre del año 2000 hay una zona libre comercio perfecta. 
En efecto, los productos originarios de los estados partes, circulan libremente en el territorio de los países integrantes del Mercosur.

El programa tenía como meta también la eliminación de las restricoiones no arancelarias que impiden o difficultan la libre circulación de bienes dentro de la zona.

Implica dos conceptos, eliminar el conjunto de restricciones no arancelarias vigentes y asumir el compromiso de no crear nuevas en el futuro.

En esta materia se llevó a cabo un proceso de armonización y desmantelamiento que se desarrollo no sin dificultades, a través del trabajo de comités especiales creados en el ámbito de la Comisión de Comercio del Mercosur (CCM), a través de los cuales se ha logrado eliminar mas del $90 \%$ de las restricciones, existiendo algunas que requieren reformas legislativas y en algunos casos modificaciones de normas constitucionales de los Estados partes.

El segundo gran paso en la dirección de la conformación de mercado común, fue el establecimiento de un arancel externo común.

La existencia de un arancel externo común (AEC) implica que las importaciones de determinados productos provenientes de un país de extrazona, tributa los mismos derechos arancelarios al ingresar a la zona integrada en cualquier punto del territorio de cualquiera de los estados miembros.

$\mathbb{E I}$ AIEC es un instrumento necesario pero no suficiente pafia la conformación de la unión aduanera y el mercado común, pero si es él elemento determinante de la existencia de un territorio aduanero unificado.

Una de las mayores dificultades para lograr acordar un arancel externo común radica en las asimetrias de las estructuras arancelarias preexistentes a la formación de la unión. las que de algún modo reflejan los diferentes patrones de industrialización que han caracterizado los modelos de desarrollo seguiclos por los paises miembros durante las últimas décadas.

La política seguida en el diseño del AEC ha sido la de alcanzar una protección efectiva, uniforme y baja.

Los criterios generales consensuados que rigen la estructura del arancel externo común son:

- No discriminar entre sectores.

- El único instrumento de protección de la actividad económica regional sera el AEC.

- Cualquier impuesto de efecto equivalente al AEC se eliminara.
- Se mantiene la posibilidad de emplear instrumentos para protegerse de las prácticas desleales de comercio exterior, para locual se cumplen las normas vigentes en la organización mundial de comercio.

- A las actividades productivaspreexistentes al proceso de integración se les reconoce un nivel de protección acorde con el diseño de la estructura arancelaria.

- Número reducido de alicuotas y baja dispersión.

El arancel externo común entró en vigencia el I de enero de 1995 a través de una decisión aprobada en la reunión del consejo del mercado común en la ciudad de ouro preto, Brasil.

Simultáneamente a la aprobación del AEC se adoptó una nueva nomenclatura, que se denominó Nomenclatura Común del Mercosur (N.C.M.).

La adopción de un arancel externo común implica como regla general que no existen aranceles nacionales diferentes entre los cuatro paises.

Para contemplar las sensibilidades de cada estado parte se establecieron excepciones al AEC por un determinado periodo de tiempo

En primer lugar, las excepciones generales que ascienden a 300 por cada pais, y en el caso particular de Paraguay 399, por el cual los aranceles nacionales tienen un cronograma de convergencia hacia el AEC que finaliza el 31 de diciembre del año 2001

En segundo lugar, se encuentran las excepciones sectoriales para bienes de capital e informática, en base a listas presentadas por los paises que tienen un plazo de convergencia previsto hasta el 31 de diciembre del año 2001 para Brasil y Argentina, y el año 2006 para Uruguay y Paraguay (AEC $14 \%$ y $16 \%$ respectivamente).

\section{Los órganos y las fuentes de derecho} del Mercosur

La conformación de la estructura orgánica del Mercosur reconoce hasta la fecha dos etapas, una primera que surge con el tratado original en el mes de marzo de 1991 y la segunda que emerge de la reunión del consejo del Mercosur en Ouro Preto en diciembre de 1994, que se regula en un documento denominado "protocolo de Ouro Preto":

\section{Personería jurídica}

A partirde Ouro Preto el Mercosur tiene personería juridica de derecho internacional en forma plena, pudien- 
mercosur, agenda para el futuro

do praclicar $ı$ dos los actos necesarios para la realización de sus objctivos.

I.a tilubaridad de la persomeria furidia la ejeree el consejo de ministros del Nercosur. sicnde yue la funcion de negociar y firmar actucedos puede ser delegalda por mandaltocipreso.

\section{Eslruclura orgánica}

El protocolo de ouro preto establece una nueva estructura organizacional de seis órganos, algunos de ellos ya contemplados en el tratado elc Asunción.

1. Fi Conscjo del Marciado Comuin (CMC).

2. Li Cirupo Mercialo Combin (CiNC)

3. La Comisión de Comercio del Mereosur (C ( MI).

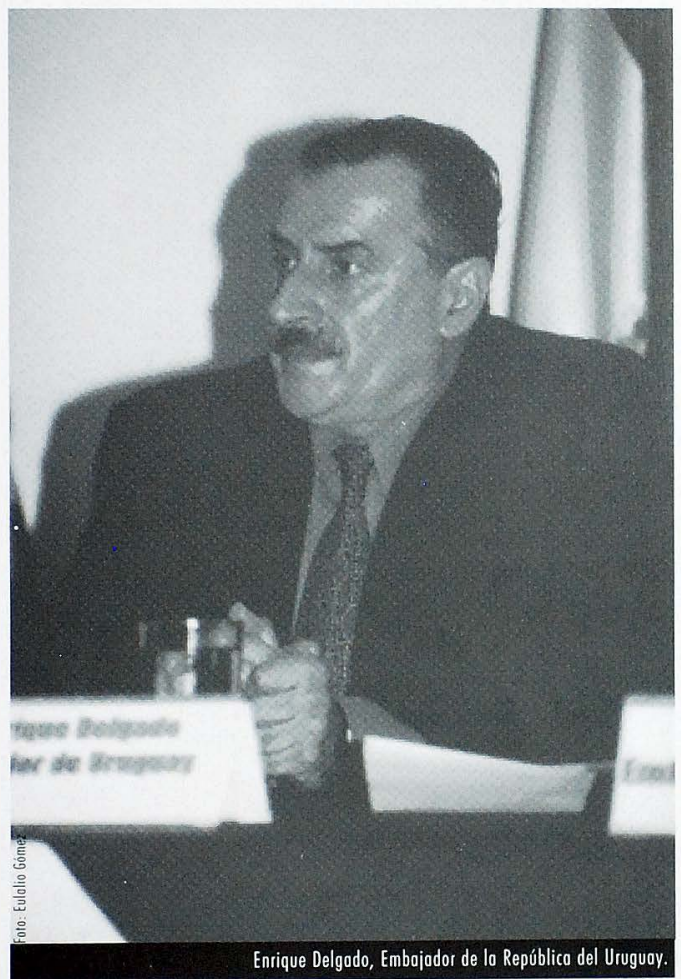

4. I.a (omisión Panlamentanal Conjunta $\left\langle C^{\circ} \cdot P^{\circ}\right)$.

i. El Foro Consulairo Economico - Soctal (l-CES)

6. La Secretaria Administrativa del Mlercosur (SAM)

En resumen las funciones son las siguientes:

E: dratade designa claramente al Consejo del Mercaldo Comuin. como el órgano supcrior del mercado comudin. correspondićndole la conduccion political del mismo.

Es el organo que loma las decisiones para ascgurar el cumplimiento de los objcivos, que inciden en la marclia del proceso

Se reume cada seis meses y está intceraclo per los ministerios de elaciones cxteriores y ele économía. Por otra parte, al menos una vez al año dehe remnirse con los presidentes de los Eslados partes (arr.ji).

Los orros 6rganos de conducción politica son las reuniones de minisuros. Las más importantes son las reuniones de ministros de economia y de presidentes de bancos centrales.

El (irupo Nercaldo (omuin - es el organno cjeculiono y lienc por ohjetion instrumemtar las clecisiones politicas (del conscjo y posec lambien capacidad de iniciativa en todas las malterais yue comprenden los objetions del tralaclo.

Del (irupo Mercade Comuin dependen 11 subgrupos de trabajo yue se reumen previamente a la reumión del grupormeraidecomutn.

Es el Grgano articulador de todas las instancias unstitucionales del acuerdo.

La comisión de comercio - es el organo encargado de asisıir al GMC. le compele velar por la aplicación de los instrumentos de politica comercial común y scguir Iodos los aspecios relacionados con el comercio intramercosur y con terceros paises. Está integrada por cualro miembros litulares y es coordinada por los ministerios de relaciones exteriores.

En el marco de la comision de comercio. se crearon diversos comilés técnicos (C.T.) encargados de analizar temas especilicos:

C.t. Sobre aranceles, nomenclatura y clasificación de mercaderías. - Se ocupa del tratimiento arancclario intra y extrazona. asi como de los lemas de nomenclaturas y clasificacion de merazderia.

$\mathrm{Cl}$ sobre asuntos aduaneros - se ocupar de lodos los lemas yue compeicen a las administraciones naciona- 
les de aduana de los Estados partes, con exclusión de las funciones establecidas para el comité técnico $\mathrm{n}^{\circ} \mathrm{I}$.

$\mathrm{Ct}$ sobre políticas públicas que distorsionan la competitividad - le compete dar cumplimiento al mandato establecido por la DEC. № 20/94, que consiste básicamente en identificar las medidas de políticas públicas que por su carácter discriminatorio puedan distorsionar las condiciones de concurrencia, clasificándolas en las categorias establecidas en la decisión mencionada, con el objetivo de elaborar propuestas para el tratamiento de las medidas a armonizar o eliminar.

Ct sobre defensa de la competencia - desarrolló el mandato establecido en la dec. $\mathrm{N}^{\circ}$ 21/94 elaborando un proyecto de estatuto de defensa de la competencia en el Mercosur, que en la actualidad está siendo incorporado a la legislación interna de los Estados miembros.

Ct sobre prácticas desleales y salvaguardias - de acuerdo a lo dispuesto en las resoluciones $n^{\circ} 108$ y $129 /$ 94. elaboró proyectos de reglamento común sobre prácticas desleales de comercio y sobre salvaguardias frente a terceros países.

Ct sobre defensa del consumidor - su mandato surge de la res. $N^{\circ} 126 / 94$, por el cual se le encomendó la preparación de un proyecto de reglamento común para la defensa del consumidor del Mercosur.

En el caso de Uruguay, cl reglamento es recogido en una ley que empezara a aplicarse a partir del $I$ de julio de este año.

La comisión parlamentaria conjunta - es el órgano de representación de los parlamentos sin perjuicio de poder remitir recomendaciones al consejo sobre cualquier tema vinculado al Mercosur. Sus principales funciones son acelerar los procedimientos internos correspondientes en los Estados partes para la pronta entrada en vigor.

El forø consuiltivo económico y social - es el órgano consultive de representación de los sectores económico sociales.

La Secretaría Administrativa - es el órgano de apoyo operativo de todo el sistema del Mercosur.

Es un órgano con sede permanente en la ciudad de Montevideo. La secretaria mantendrá el archivo oficial de la documentación del Mercosur; editará un boletín oficial del Mercosur y efectuará las traducciones auténticas del Mercosur. También informará regularmente a los Estados partes de las medidas implementadas por cada país para incorporar a sus ordenamientos jurídicos las normas emanadas de los órganos. Estará a cargo de un director electo por el GMC y designado por el consejo con mandato por dos ańos.

\section{Las fuentes del derecho sub-regional}

Se distinguen entre las fuentes de derecho el tratado de Asunción

Los protocolos o acuerdos celebrados en el marco del mismo y los actos emanados de los órganos comunitarios.

Por último, debemos agregar los laudos de los tribunales arbitrales, que se expiden en el marco del protocolo de Brasilia para la solución de controversias.

\section{Sistema de toma de decisiones y aplicación interna}

Las normas del Mercosur son todas obligatorias a título expreso, ya sean estas decisiones del consejo; reso. luciones del grupo mercado común o directivas de la comisión de comercio y las mismas son adoptadas por consenso y con la presencia de todos los Estados partes.

Una vez aprobadas por los órganos del Mercosur es obligatorio su incorporación al derecho interno de los Estados partes.

\section{Solución de controversias}

Se mantiene como sistema de solución de controversias el protocolo de Brasilia del 17 de diciembre de 1991, con el agregado de que todas las controversias que planteadas sobre la interpretación, aplicación o incumplimiento de las directivas de la comisión de comercio también se incluyen en el mismo sistema.

Asimismo, en el anexo al protocolo de ouro preto se establece un procedimiento de características prejurisdiccionales sobre las reclamaciones presentadas a la Comisión de Comercio.

Dichas reclamaciones pueden ser originadas por las secciones nacionales de la comisión de comercio o por particulares.

Por último, en el artículo 44 se establece que antes de culminar el proceso de convergencia del arancel externo común, los Estados partes adoptarán un sistema permanente de solución de controversias. 
mercosur, agenda para el futuro

\section{El relanzamiento del Mercosur}

En el de desarrollo del mercado comuin del sur hal sido muy importante el impulso que brindan los jeles de Estado para dotar al proceso de nucvos estimulos en los periodos en que la dinamica se aletargal.

Precisamente. el denominado relanzamiconto del Mercosur o las relormas de tereera generacion que se estan gestando contemporancamente. son producto de una decision politica adoptada por los jeles de Estado de los Estidos partes.

Habiendo logrado al finalizar cl milenio una union aduanera casi perlecla. el desafio para la próxima cenuıria es lograr la conformación de un mercado común.

El principal punto de la agenda hacia el futuro del Mercosur es profundizar él esquema de integración para alcanzar la libre circulacion de bienes. laciores productivos. capilall y trabajo.

En este semtido. los presidentes del M.lereosur. que asumen nuevos mandatos durante 1999 y en el primer trimestre del año 20(0). toman la decision de relanzar el proceso impulsando una lerecra generación de reformas.

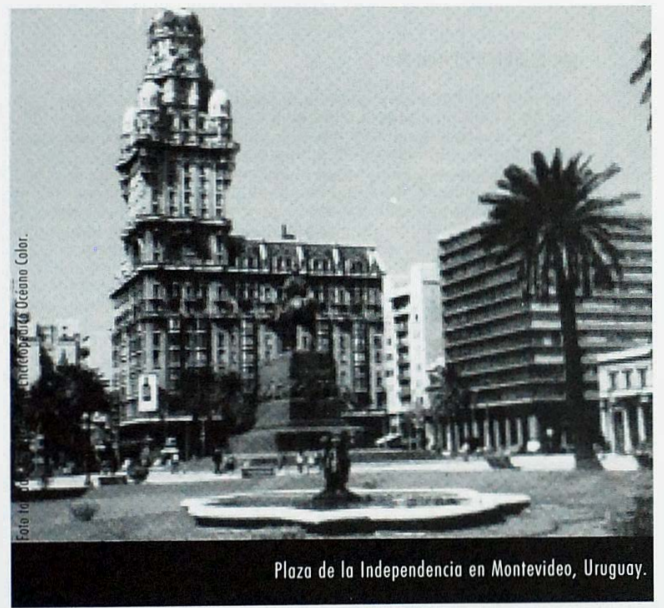

Un nucvo impulso político para acelcrar y profundizar la realización de los objclivos trazados en el Mercosur. Un proceso que ya ticne en los primeros meses del año 2000 señales de avances muy concrelos.

En primer lugar se acordo en el mes de abril pasado después de varios años de negociación. un régimen común para el secior automotor que establece un sistcma de convergencia hacia un arancel externo común y también establece las condiciones para la libre circulación de los producios del secior

Por otro lado. en el mes de mayo se acordo las bases para cslablecer un maıco regional cnergético común que implica la coordinación de las politicas energélıcas. la armonización de los marcos regulatorios y por último. la liberalización de los producios energélicos como gas. electricidad y combustibles.

Pero quizas lo mas importante fuc el anuncio de la formalizaciondel denominado "pequenom mastrich". que implica el primer esfuerzo serio que se realiza paral la coordinacion de politicas macroeconomicas.

En el mes de septicmbre los cuatro Estados partes publicarin estadisticis calculadas sobre una base comparable que permitiri eotcjar fa situación liscal de caida uno de los micmbros del bloque.

Una vez realizada esta publicacion. se preve que en el mes de marzo próximo, los Estados adoptarán las melas que deberán cumplir para la convergencia de sus principa!es variables economicas, que comprende. entre otras. déficit fiscal. inliación y limite de deuda publica.

Este es un proceso que está en curso, nuevas señales surgirán durantc la reunion del consejo que se rcalizara en Buenos Aires en las proximas semanas y en la reunión que se realizará a lin de año en Brasil.

No obstante cllo. podemos ir identificindo los temas que integran la agenda del Mereosur hacia el fuluro.

\section{"Aggiornamento" institucional}

Existe una opiniòn generalizada a nivel académico en cuanto a que la estructura institucional de un sistema de integracion yue précende consoliclar una unión aduatnera ! un merade eombin. requiere de un conjunto de 
órganos, intèrgubernamentales. técnicos y comunitarios.

Disponer de la capacidad real para conducir y administrar un proceso tan complejo como el Mercosur no se puede sustentar exclusivamente en mecanismos intergubernamentales.

Es necesario incorporar mecanismos de apoyo técnico y componentes de naturaleza comunitaria.

La condición principal para el éxito de un mercado común consistirá en qque sea duradero, que se caracterice por su estabilidad y permanencia y que en su proceso formativo no se incurira en retrocesos y dilaciones en el desmantelamiento de los obstáculos a los intercambios, ni se desacompasen la armonización de los instrumentos y la coørdinación de políticas macroeconómicas y sectoriales.

Para ello se requiere la adopción de un sistema " direccional ", esto es, la existencia de instituciones comunitarias con poderes para dirigir el proceso

Es necesario aumentar la capacidad de gestión en el proceso, y.a que las cancillerías y los ministerios del área económica no tienen los recursos humanos necesarios para abocarse a esta etapa del proceso.

Los desafios futuros demandan abarcar otras actividades que no se limiten al campo económico, ya que las definiciones de las prioridades no se realizarán con criterios exclusivamente de carácter técnico- económico.

En otras palabras, el ritmo del tratamiento de los temas sociales. educacionales, laborales y medioambientales tiene que ser igual a los de naturaleza económica.

En este sentido, uno de las medidas inmediatas podría ser ampliar la competencia de la secretaria administrativa incorporándole funciones de contenido técnico.

\section{Fortalecer el control de legalidad}

El establecimiento a mediano plazo de un mercado común suscita inevitablemente controversias en materia de interpretación jurídica, de incumplimientos de compromisos pactados y de aplicación de políticas incompatibles con los objetivos propuestos y reñidas, con el principio de "pacta sunt senranda".

Por lo tanto. se requiere de un régimen jurisdiccional lo más perfecto posible.

El tratado de Asunción no creo mecanismos avanzados en esta materia. El protocolo de Brasilia, y las normas especiales y complementarias relativas a la comisión de comercio. Introdujeron mecanismos mas perfeccionados, pero todavia insuficientes.

Un mecanismo arbitral que cumplió su objetivo en la consolidación de la zona de libre comercio y en el per-

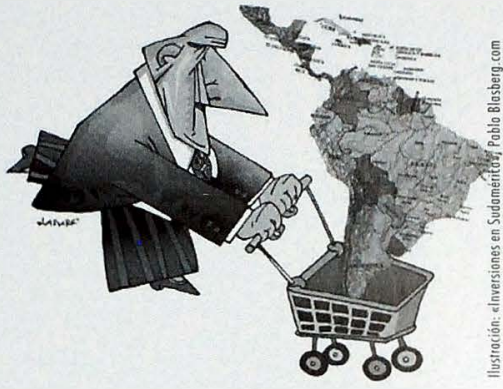

feccionamiento de la unión aduanera pero que reiteramos, es insuficiente si pretendemos avanzar hacia un mercado común.

Es necesario la creación de un órgano jurisdiccional con competencias explicitas cuyas decisiones tengan la máxima jerarquía jurídica y no estén acotadas por una excesiva retención de atribuciones de los poderes públicos nacionales.

Los dictámenes de ese órgano, como ocurre con los de la corte europea de justicia, debieran ser de aceptación obligatoria y de aplicación automática e inmediata.

Como paso intermedio se considera que es posible establecer un mecanismo independiente complementario, con el cometido de registrar regularmente las medidas legales y administrativas que adopten las autoridades de los Estados partes y supervisar su adecuación a la normativa comunitaria y de alertar a los órganos del sistema sobre eventuales casos de incumplimiento.

Esta tarea de fiscalización no iría en desmedro de las autonomías nacionales, sino que, por el contrario, contribuiria a disciplinar acciones dispersas y a corregir errores de interpretación o de aplicación en los diversos niveles burocráticos.

\section{Las asimetrías}

Existe un principio esencial e histórico en la integración latinoamericana que establece que las disparidades de desarrollo y de capacidad económica entre los países que participan en un proceso de integración tienen que ser considerados en forma especial y dar lugar a tratamientos diferenciales o excepcionales. Este mismo criterio es reconocido por la OMC y también esta en la mesa de negociación del ALCA. 
mercosur, agenda para el futuro

Sin embargo, este principio fue considerado e $n$ forma parcial y limilada en el tratado de Asunción. estableciéndose tratamientos diferenciales sólo en el campo arancelario no abarcando otros aspecios de las relaciones económicas comunitarias.

Esta concepción puede ser admisible en la conformación de una \%ona de libre comercio o en una unión aduanera, pero no en la perspectiva de un mercado común donde se requiere extender el campo de acción de este principio.

En otras palabras, pasar del principio de la reciprocidad plena a la reciprocidad efectiva, que tiende a equiparar las desigualdades mediante una aceptable equivalencia de resultados.

\section{Las inversiones}

Un desarrollo armónico y equilibrado del nuevo espacio multinacional se asocia al criterio de la distribución equitativa de los beneficios de la integración.

Los beneficios no sólo comprenden las facilidades que se obtienen con el acceso a un mercado preferencial sino que más importante que lo anterior, es la posibilidad de atraer inversiones foráneas direcias.

Podría afirmarse que el termómetro de las ganancias del comercio de mercancias en un sistema integrado, serán la cantidad y la modernidad tecnológica de los bienes de capital que se incorporen a la estructura productiva de cada Estado.
En particular. en el caso de los pequeios Estados. es la razón de ser de su participación en un meriado ampliado.

Por lanto es fundamental que se establezca rápıdamente una politica común de incentivos o una efectiva coordinación de las medidas nacionales para la atraeción de inversiones, ya que de no ser asi, las inversiones se orientarín a ayuel pais yue más otorgue y altermativa o complementariamente. hacia el pais con el meicado más seductor.

Este tiltimo aspecto, el factor dimensional es gravitante, porque la conformación del Mereado Común real lleva tiempo. aun después de haherse cumplido la libcracion comercial.

La experiencia de los últimos años no ha sido buena ya cue el otorgamiento de incentivos a la inversión foránea. de manera unslateral y descoordinada llevó a los Estados partes a una competencia desordenada para conseguir radicaciones de establecimientos productivos en sus territorios por la via de mayores y eventualmente exageradas ventajas a los inversores.

En definitwa y como conclusión final, la inciativa del relanzamiento del Nercosur es la ratificación de la voluntad politica de los paises miembros del bloque cle consolidar y profundizar el proceso de integración.

Una respuesta a las incertidumbres que plantea el mercado mundial en donde predominan cada vez más las práticas proteccionistas, lo que provoca el aumento de la brecha entre los paises ricos y los que se encuentran en vias de desarrollo.

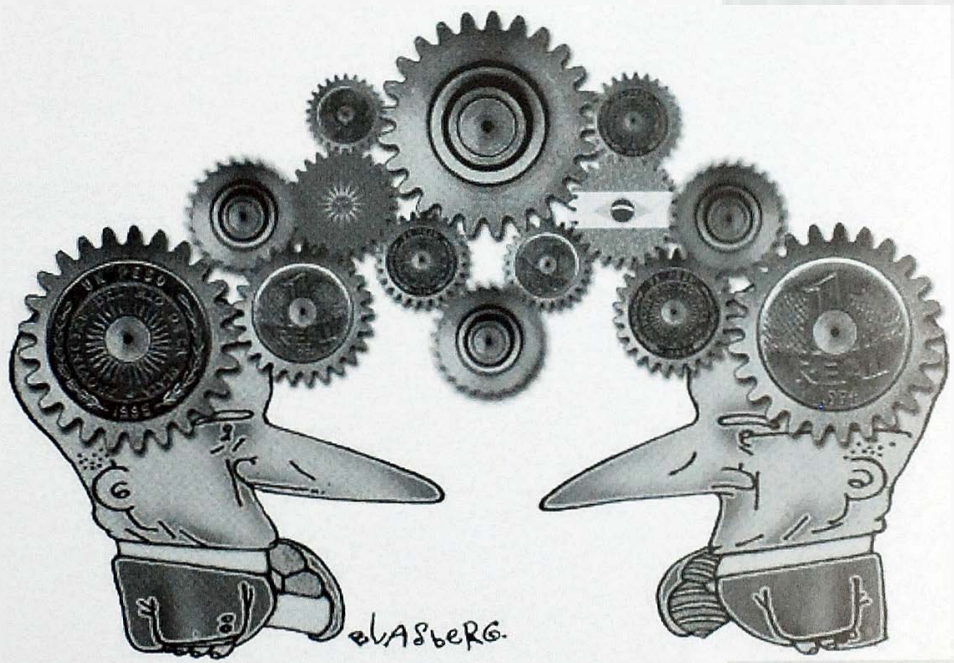

Ilustración: "Mercosurn, Poblo Blosberg 